\section{Laser Direct-Write Processing}

\section{Craig B. Arnold and Alberto Piqué, Guest Editors}

\begin{abstract}
Direct-write techniques enable computer-controlled two- and three-dimensional pattern formation in a serial fashion. Among these techniques, the versatility offered by laser-based direct-write methods is unique, given their ability to add, remove, and modify different types of materials without physical contact between a tool or nozzle and the material of interest. Laser pulses used to generate the patterns can be manipulated to control the composition, structure, and even properties of individual three-dimensional volumes of materials across length scales spanning six orders of magnitude, from nanometers to millimeters. Such resolution, combined with the ability to process complex or delicate material systems, enables laser direct-write tools to fabricate structures that are not possible to generate using other serial or parallel fabrication techniques. The goal of the articles in this issue of MRS Bulletin is to illustrate the range of materials processing capabilities, fundamental research opportunities, and commercially viable applications that can be achieved using recently developed laser direct-write techniques. We hope that the articles provide the reader with a fresh perspective on the challenges and opportunities that these powerful techniques offer for the fabrication of novel devices and structures.
\end{abstract}

\section{Introduction}

From the earliest work on laser interactions with materials, direct-write processes have been important and relevant techniques to modify, add, and subtract materials for a wide variety of systems and for applications such as metal cutting and welding. In general, direct-write processing refers to any technique that is able to create a pattern on a surface or volume in a serial or "spot-by-spot" fashion. This is in contrast to lithography, stamping, directed self-assembly, or other patterning approaches that require masks or preexisting patterns. At first glance, one may think that direct-write processes are slower or less important than these parallelized approaches. However, direct-write allows for precise control of material properties with high resolution and enables structures that are either impossible or impractical to make with traditional parallel techniques. Furthermore, with continuing developments in laser technology providing a decrease in cost and an increase in repetition rates, there is a plethora of applications for which laser direct-write (LDW) methods are a fast and competitive way to produce novel structures and devices. This issue of MRS Bulletin seeks to assess the current status and future opportunities of LDW processes in the context of emerging applications.

There are many types of direct-write techniques used in science and engineering. ${ }^{1}$ For instance, previous MRS Bulletin issues discussed topics such as inkjet printing (November 2003) and focused ion-beam processing (February 2000, July 2001, and December 2005). In the most classical sense, engraving or milling can be considered a direct-write process, since a tool or stylus makes contact with a surface and is moved in a desired pattern to produce a feature. The coupling of a highpowered laser with direct-write processing enables similar features to be produced without requiring physical contact between a tool and the material of interest. Because of this, few techniques share the versatility of LDW in adding, subtracting, and modifying different types of materials over many different length scales, from the nanometer to the millimeter scale.
In LDW, the beam is typically focused or collimated to a small spot (in industrial processes, this "small" spot can be several millimeters in diameter). Patterning is achieved by either rastering the beam above a fixed surface or by moving the substrate or part within a fixed beam. An important feature of LDW is that the desired patterns can be constructed in both two and three dimensions on arbitrarily shaped surfaces, limited only by the degrees of freedom and resolution of the motion-control apparatus. In this manner, LDW can be considered a "rapid prototyping $^{\prime 2-4}$ tool, because designs and patterns can be changed and immediately applied without the need to fabricate new masks or molds.

The key elements of any LDW system can be divided into three subsystems: (1) laser source, (2) beam delivery system, and (3) substrate/target mounting system (see Figure 1). At the heart of any LDW process is the laser source. Typical experiments and applications use anywhere from ultrafast femtosecond-pulsed systems to continuous-wave systems employing solid-state, gas, fiber, semiconductor, or other lasing media. In choosing an appropriate source, one must consider the fundamental interactions of lasers with the material of interest. This requires knowledge of the pulse duration, wavelength, divergence, and other spatial and temporal characteristics that determine the energy absorption and the material response. In beam delivery, there are a variety of ways to generate a laser spot, including fixed focusing objectives and mirrors, galvanometric scanners, optical fibers, or even fluidic methods such as liquid-core waveguides ${ }^{5}$ or water jets. ${ }^{6}$ The choice depends on the application demands, for instance, the required working distances, the focus spot size, or the energy required. The ultimate beam properties will be determined by the combination of laser and beam delivery optics. Finally, the substrate mounting is done in accordance with experimental or industrial requirements and can be manipulated in multiple directions to achieve a desired result. Robotics and active feedback control, on either the substrate or beam delivery optics, can add further design flexibility to the technique.

There is a vast range of LDW processes. For the purposes of this issue, we categorize them into three main classes: laser directwrite subtraction (LDW-), where material is removed by ablation; laser direct-write modification (LDWM), where material is modified to produce a desired effect; and laser direct-write addition $(\mathrm{LDW}+)$, where material is added by the laser. 


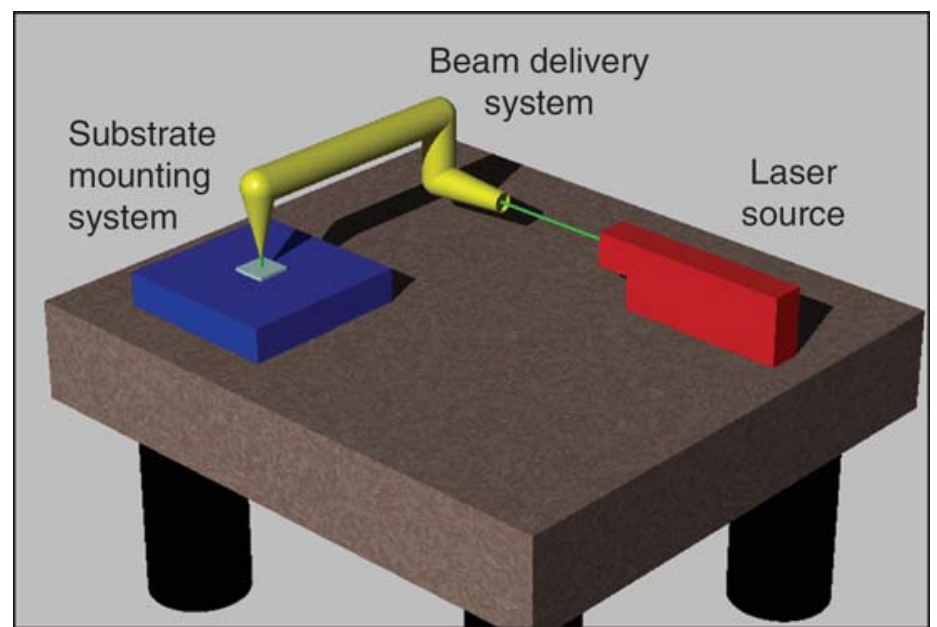

Figure 1. Schematic illustration of a laser direct-write system. The basic components of an LDW system are (left to right) a substrate mounting system, a beam delivery system, and a laser source. Motion control of either the beam delivery system or the substrate mounting system is typically accomplished using computer-assisted design and manufacturing (CAD/CAM) integrated with the laser source.

\section{Laser Direct-Write Subtraction (LDW-)}

LDW - is the most common type of laser direct-write. In general, this entails processes that result in photochemical, photothermal, or photophysical ablation on a substrate or target surface, directly leading to the features of interest. ${ }^{7}$ Common processes include laser scribing, cutting, drilling, or etching to produce relief structures or holes in materials in ambient or controlled atmospheres. ${ }^{8}$ Industrial applications using this technique range from high-throughput steel fabrication, to inkjet and fuel-injection nozzle fabrication, ${ }^{9,10}$ to high-resolution manufacturing and texturing of stents or other implantable biomaterials. ${ }^{11}$ At a smaller scale, inexpensive benchtop laser cutting and engraving systems can be purchased by the hobbyist or small company for artistic and architectural renderings. More recent developments in LDW- include chemically assisted techniques such as laser-drilling ceramics or biomaterials and laser-induced backside wet etching (LIBWE) of glass. ${ }^{12,13}$ In fact, one may also consider laser cleaning to be a controlled LDW- process. $^{14}$

The fundamental interactions leading to material removal can be thermal or athermal, depending primarily on the material/environment characteristics and the pulse duration of the laser. These interactions have a direct effect on the quality of the resulting features. For instance, a heataffected zone (HAZ) tends to occur in the vicinity of thermally removed material. This region has structures and properties that can differ from the bulk material and can exhibit additional surface relief. Either of these effects may be beneficial or detrimental, depending on the application. In contrast, athermal and multiphoton absorption processes caused by ultrafast lasers can reduce the formation of a HAZ and enable features smaller than the diffraction limit. ${ }^{15,16}$

\section{Laser Direct-Write Modification (LDWM)}

In LDWM, the incident laser energy is usually not sufficient to cause ablative effects but is sufficient to cause a permanent change in the material properties. Typically, these processes rely on thermal modifications that cause a structural or chemical change in the material. A common example of such processes is the rewritable compact disc, in which a diode laser induces a phase transition between crystalline and amorphous material. ${ }^{17}$ In industrial applications, one may consider laser cladding, where a surface layer different from the bulk material is produced through melting and resolidification, ${ }^{18}$ or solid free-form fabrication (SFF) approaches such as selective laser sintering (SLS), ${ }^{19}$ as important modifying processes that would fall under the umbrella of LDWM.

Many LDWM applications require a specific optical response in the material of interest beyond simple thermal effects. Optically induced defects or changes in mechanical properties can lead to many non-ablative material modifications. For instance, photoresists respond to light by breaking or reforming bonds, leading to pattern formation in the material. Alternatively, LDW can cause defects in photoetchable glass ceramics ${ }^{20}$ or other optical materials through single- and multiphoton mechanisms ${ }^{21}$ enabling novel applications in optical storage, ${ }^{22}$ photonic devices, ${ }^{23,24}$ and microfluidics. ${ }^{25}$

\section{Laser Direct-Write Addition (LDW+)}

LDW + is perhaps the most recent of the laser direct-write processes. In this technique, material is added to a substrate using various laser-induced processes. Many techniques are derived from laserinduced forward transfer (LIFT), where a sacrificial substrate of solid metal is positioned in close proximity to a second substrate to receive the removed material. ${ }^{26}$ The incident laser is absorbed by the material of interest, causing local evaporation. This vapor is propelled toward the waiting substrate, where it recondenses as an individual three-dimensional pixel, or voxel, of solid material. Such an approach has found important use in circuit and mask repair and other small-scale applications where one needs to deposit material locally to add value to an existing structure. This general technique has significant advantages over other additive direct-write processes, in that these laser approaches do not require contact between the depositing material and a nozzle, and can enable a broad range of materials to be transferred. Variations on the general LIFT principle allow liquids, inks, and multiphase solutions to be patterned with computer-controlled accuracy for use in a variety of applications such as passive electronics or sensors. ${ }^{27,28}$

Alternatively, LDW + techniques can rely on optical forces to push particles or clusters into precise positions, ${ }^{29}$ or on chemical changes in liquids and gases to produce patterns. For instance, laser-induced chemical vapor deposition, ${ }^{30}$ or multiphoton polymerization schemes of liquid photoresists, ${ }^{31}$ can be used to fabricate three-dimensional stereographic patterns. Examples of this have been demonstrated and show promise for many applications such as fabricating photonic structures or biological scaffolding.

\section{Highlights of Articles in This Issue}

The breadth of LDW processing makes it impossible to cover the entire topic in a single MRS Bulletin issue. The situation is further complicated by the fact that many LDW processes have either grown into mature fields in and of themselves (laser cutting, welding, etc.) or provide ample topical material for an independent $M R S$ Bulletin issue (solid free-form fabrication, 
laser cleaning, fiber laser development, etc.). Therefore, we restrict ourselves to LDW processes developed in the past few years that provide opportunities for growth beyond the traditional industrial applications. Clearly, this coverage is not exhaustive, and there are many other LDW approaches that could be discussed. However, the reader of this issue should get a good sense of the possibilities of LDW.

We have purposely avoided focusing on femtosecond laser techniques because the August 2006 issue of MRS Bulletin on "Ultrafast Lasers in Materials Science" covered this topic in detail, including some LDW approaches. Femtosecond lasers are very important in many of the newer LDW approaches, as they enable higher-resolution features, detailed control over structures, and unique nonthermal processing capabilities. The reader is referred to the earlier MRS Bulletin for more information.

In the first article in this issue, by Grigoropoulos et al., we look at approaches for LDW - that can produce features significantly smaller than the diffraction limit of light. In this approach, a near-field phenomenon is utilized that radiates the electromagnetic field to the region directly below an atomic force microscope (AFM) tip, removing material in an area as small as $10 \mathrm{~nm}$. Rastering is controlled by the motion of the AFM stage, and various features can be produced. By coating the tip with metal, deposition on the nanoscale by LDW+ can also be accomplished.

Two articles on LDW + follow. In the first, by Arnold et al., we look at LDW + of complex materials, including biological materials such as cells and proteins, electrochemical materials for energy storage, and even complete semiconductor devices. The approach relies on laser forward transfer of multicomponent suspensions composed of different liquid and/or solid materials, which allows for deposition without harming sensitive material properties. This technique can be further combined with LDWM or LDW - to perform postdeposition processing with the same tool. Next, Stuke et al. turn our attention to the $\mathrm{LDW}+$ fabrication of threedimensional structures such as cages for trapping particles and photonic structures for guiding light. In this technique, a laserbased chemical vapor deposition method produces highly conductive, freestanding metal features. One particularly interesting application is the production of carbon nanotubes and wires in an LDW fashion.
One of the key advantages of LDWM for material modification, in contrast to many parallel approaches, is the ability to apply a unique photon flux at each laser spot. Livingston and Helvajian discuss a real-time sequencing approach that programs the incident laser pulses to enable distinctive, site-selective processes. This can be applied to photomodifiable glasses and glass ceramics to produce complex features that would be difficult or impossible to make by other methods.

Finally, we look toward the future of LDW with Sugioka et al., who discuss the global industrial aspects of LDW processing. Cutting-edge applications in Europe, the United States, and Japan are discussed, with implications for long-term growth.

\section{Summary}

One of the unique attributes of laser direct-write processes is that the general methodology enables a complete range of materials processing in order to fabricate devices and structures. These techniques make it possible for a single tool, located in the research lab or on the factory floor, to design and build an entire part with precise control over the composition, structure, and properties on each laser pulse.

Although that vision of LDW has not been reached, the articles in this issue of MRS Bulletin cover additive, subtractive, and modification methods and demonstrate a commercially viable technology with abundant opportunities for fundamental research that will continue to push advancements in the field. Ideally, these approaches lend themselves to rapid prototyping and small-scale production where unique features are needed for a desired application. However, they also find important implementation in commercial ventures where high-repetition-rate, multibeam laser systems allow LDW to compete with parallel processing in speed and cost. The impact of LDW processes in advanced applications will continue to grow as new methods and technologies become available and the demands for more precise control over material structures and properties are met.

\section{References}

1. See for instance, D.B. Chrisey, Science 289 (2000) p. 879; A. Piqué and D.B. Chrisey, eds., Direct-Write Technologies for Rapid Prototyping Applications (Academic Press, San Diego, 2002).

2. C.C. Kai, L.K. Fai, and L.C. Sing, Rapid Prototyping: Principles and Applications (World Scientific, London, 2004).
3. P.K. Venuvinod and W. Ma, Rapid Prototyping: Laser-Based and Other Technologies (Kluwer Academic, Norwell, MA, 2003).

4. A. Piqué and D.B. Chrisey, eds., Direct-Write Technologies for Rapid Prototyping Applications (Academic Press, San Diego, 2002).

5. K.W. Gregory and R.R. Anderson, IEEE J. Quant. Elect. 26 (1990) p. 2289.

6. P. Couty, F. Wagner, and P. Hoffmann, Opt. Eng. 44068001 (2005).

7. D. Bäuerle, Laser Processing and Chemistry, 3rd Ed. (Springer, Berlin, 2000).

8. W.M. Steen and K. Watkins, Laser Materials Processing, 3rd Ed. (Springer, Berlin, 2003).

9. H. Endert, R. Patzel, and D. Basting, Opt. Quant. Elect. 27 (1995) p. 1319.

10. M.C. Gower, Opt. Exp. 7 (2000) p. 56.

11. A. Kureil and N.B. Dahotre, J. Biomed. Appl. 5 (2005) p. 5.

12. X. Ding, Y. Kawaguchi, H. Niino, and A. Yabe, Appl. Phys. A 75 (2002) p. 641.

13. G. Kopitkovas, T. Lippert, C. David, A. Wokaun, and J. Gobrecht, Microelectron. Eng. 67 (2003) p. 438.

14. V. Tornari, V. Zafiropulos, A. Bonarou, N.A. Vainos, and C. Fotakis, Opt. Lasers Eng. 34 (2000) p. 309.

15. P.P. Pronko, S.K. Dutta, J. Squier, J.V. Rudd, D. Du, and G. Mourou, Opt. Commun. 114 (1995) p. 106.

16. M. Lenzner, J. Kruger, W. Kautek, and F. Krausz, Appl. Phys. A 68 (1999) p. 369.

17. J. Feinleib, J. deNeufville, S.C. Moss, and S.R. Ovshinsky, Appl. Phys. Lett. 18 (1971) p. 254. 18. R. Vilar, J. Laser App. 11 (1999) p. 64.

19. D.L. Bourell, H.L. Marcus, J.W. Barlow, and J.J. Beaman, Int. J. Powder Metall. 28 (1992) p. 369 .

20. T.R. Dietrich, W. Ehrfeld, M. Lacher, M. Kramer, and B. Speit, Microelectron. Eng. 30 (1996) p. 497.

21. K. Itoh, W. Watanabe, S. Nolte, and C.B. Schaffer, MRS Bull. 31 (8) (2006) p. 620.

22. E.N. Glezer, M. Milosavljevic, L. Huang, R.J. Finlay, T.H. Her, J.P. Callan, and E. Mazur, Opt. Lett. 21 (1996) p. 2023.

23. P.R. Herman, R.S. Marjoribanks, A. Oettl, K. Chen, I. Konovalov, and S. Ness, Appl. Surf. Sci. 154 (2000) p. 577.

24. A. Zoubir, M. Richardson, C. Rivero, A. Schulte, C. Lopez, K. Richardson, N. Ho, and R. Vallee, Opt. Lett. 29 (2004) p. 748.

25. Y. Cheng, K. Sugioka, and K. Midorikawa, Opt. Lett. 29 (2004) p. 2007.

26. J. Bohandy, B.F. Kim, and F.J. Adrian, J. Appl. Phys. 60 (1986) p. 1538.

27. D.B. Chrisey, A. Piqué, J. Fitz-Gerald, R.C.Y. Auyeung, R.A. McGill, H.D. Wu, and M. Duignan, Appl. Surf. Sci. 154 (2000) p. 593.

28. A. Pique, R.C.Y. Auyeung, J.L. Stepnowski, D.W. Weir, C.B. Arnold, R.A. McGill, and D.B. Chrisey, Surf. Coat. Technol. 163 (2003) p. 293.

29. M.J. Renn and R. Pastel, J. Vac. Sci. Tech. B 16 (1998) p. 3859

30. S.D. Allen, J. Appl. Phys. 52 (1981) p. 6501. 31. J. Serbin, A. Egbert, A. Ovsianikov, B.N. Chichkov, R. Houbertz, G. Domann, J. Schulz, C. Cronauer, L. Frohlich, and M. Popall, Opt. Lett. 28 (2003) p. 301. 


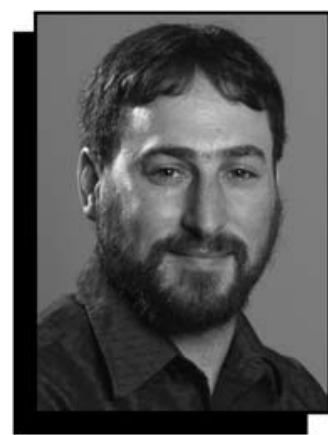

Craig B. Arnold

Craig B. Arnold, Guest Editor for this issue of MRS Bulletin, is an assistant professor of mechanical and aerospace engineering at Princeton University with a joint appointment in the Princeton Institute for Science and Technology of Materials (PRISM) and affiliations in electrical engineering and the Princeton Environmental Institute. He received his BS degree in physics and math from Haverford College in 1994, his PhD degree in physics from Harvard University in 2000, and worked as a National Research Council postdoctoral associate prior to joining the faculty of Princeton in 2003. His current research focuses on laser processing and transport in materials, with a particular focus on energy storage, biomaterials, and photonic materials.

Arnold received a Young Investigator Award from the Office of Naval Research in 2005 and a National Science Foundation CAREER Award in 2006. He currently serves as chair of the MRS Academic Affairs Committee and as an associate editor of the Journal of Laser Micro/Nanoengineering.

Arnold can be reached at Princeton University, Department of Mechanical and Aerospace

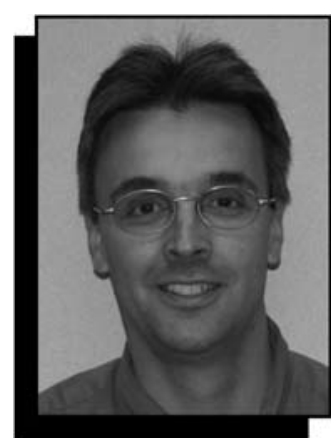

Alberto Piqué

Engineering, Engineering Quadrangle, 1 Olden St., Princeton, NJ 08544 USA; tel. 609-258-0250 and e-mail cbarnold@princeton.edu.

Alberto Piqué, Guest Editor for this issue of MRS Bulletin, is head of the Electronic and Optical Materials and Devices Section in the Materials Science Division at the U.S. Naval Research Laboratory (NRL). Piqué holds BS and MS degrees in physics from Rutgers University and $\mathrm{ahD}$ degree in materials science and engineering from the University of Maryland. His group at the NRL pioneered the use of laser-based direct-write techniques for rapid prototyping of electronic, sensor, and micropower generation devices. His research interests include the study of processes involving laser-material interactions, particularly those relevant to pulsed laser deposition and laser forward transfer as applied to the fabrication of thin films, multilayers, and embedded structures.

In addition to coediting the book DirectWrite Technologies for Rapid Prototyping Applications, Piqué has more than 130 scientific publications and holds 15 U.S. patents in laser materials processing. He is also

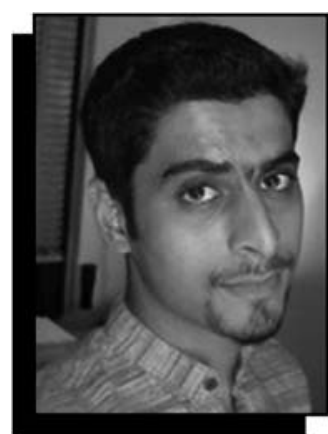

Anant Chimmalgi

an associate editor for the Journal of Laser Micro/ Nanoengineering.

Piqué can be reached at the Naval Research Laboratory, Code 6364, 4555 Overlook Ave. SW, Washington, DC 20375 USA; tel. 202-767-5653 and e-mail pique@ nrl.navy.mil.

Anant Chimmalgi is a system design engineer at KLA Tencor Corp. He received his $\mathrm{BE}$ degree from the National Institute of Technology in Surathkal, India, and his MS and PhD degrees from the Department of Mechanical Engineering at the University of California, Berkeley (2003 and 2005, respectively). Chimmalgi's current research interests include bright-field wafer inspection technologies, semiconductor yield management, nanofabrication technologies, nanoscale heat transfer, nanoparticle manipulation, scanning probe microscopy, and ultrashort laser phenomena.

Chimmalgi can be reached at 5144 Etcheverry Hall, University of California, Berkeley, CA 94720-1740 USA; tel. 510-642-1006 and e-mail anchip@ocf.berkeley.edu.

Günter Fuhr is executive director of the Fraunhofer-Institut für Biomedizinische Technik (IBMT) in St. Ingbert,

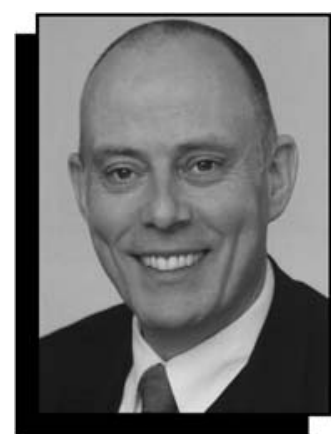

Günter Fuhr

Germany. He earned his Dipl.-Ing. degree in electrical engineering at Technische Universität Dresden in 1975. Fuhr received his Dr. rer. nat. degree in biophysics in the field of plant physiology from Humboldt-Universität zu Berlin (HUB) in 1981. He also earned his Habilitation degree in the field of cell biology and biophysics from HUB in 1985.

Fuhr became a full professor at the Institute of Biology at HUB in 1993. From 1994 to 1996, he was vice dean of the faculty of Mathematics and Natural Sciences I at HUB. In 2000, Fuhr became the founding director of the Center of Biophysics and Bioinformatics at HUB. He became a full professor and chair of the Biotechnology and Medical Technology Department at the Medical Faculty of the Universität des Saarlandes in 2001 and executive director of branches in Sulzbach/Saar, Potsdam, Berlin, and Shenzhen, China, that same year. In 2003, Fuhr was the founding director of the Research Cryobank within the Center for Cryobiotechnology (EUROCRYOSaar) in Sulzbach/Saar.

Fuhr can be reached at Fraunhofer-Institut für Biomedizinische

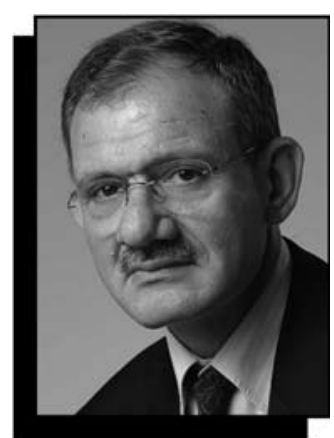

Costas P. Grigoropoulos

Technik, Ensheimer Strasse 48, D-66386 St. Ingbert, Germany; tel. 49-6894-980-100, fax 496894-980-110, and e-mail guenter.fuhr@ibmt. fraunhofer.de.

Costas P. Grigoropoulos is a professor in the Department of Mechanical Engineering at the University of California, Berkeley, and on the materials science/engineering faculty of the Environmental Energy Technologies Division at Lawrence Berkeley National Laboratory.

Grigoropoulos received Diploma degrees in naval architecture and marine engineering and in mechanical engineering from the National Technical University of Athens, Greece. He holds MSc and PhD degrees in mechanical engineering from

Columbia University.

His current research interests are in micro/ nanoengineering, laser materials processing and micro/nanomachining, microscale and nanoscale diagnostics for chemical analysis, phase transformations in semiconductors and electronic materials, laser-driven thin-film crystal growth for applications in microelectronic devices, nanofluidics, microscale fuel cells, catalytic microreactors, hydrogen 


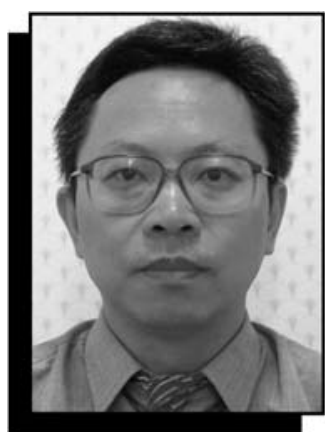

Bo $\mathrm{Gu}$

storage, thermal management in microdevices, and transport diagnostics in MEMS. Grigoropoulos is a fellow of ASME.

Grigoropoulos can be reached at the University of California, Berkeley, Department of Mechanical Engineering, 6177 Etcheverry Hall, Berkeley, CA 94720-1740 USA; tel. 510-642-2525, fax 510-642-6163, and e-mail cgrigoro@ me.berkeley.edu.

Bo $\mathrm{Gu}$ is senior principal scientist at GSI Group Inc. He has been working in the fields of lasers and laser applications in industry for more than 20 years.

In the early 1990s, Gu worked on developing excimer lasers for semiconductor lithography. $\mathrm{Gu}^{\prime}$ s group was the first to develop a $\mathrm{CO}_{2}$ laser system for microvia drilling for the microelectronics industry. $\mathrm{He}$ has studied and developed many industrial micromachining applications using diode pumped solid-state, excimer, and $\mathrm{CO}_{2}$ lasers. His current interests include the development and industrial applications of new types of lasers. Gu has published many papers on lasers and laser applications, organized and chaired various conferences, and has more than

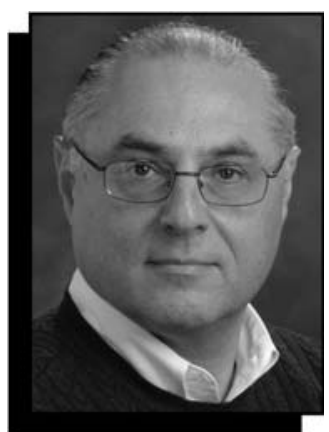

Henry Helvajian

20 patents or pending patents.

$\mathrm{Gu}$ can be reached at GSI Group, 60 Fordham Road, Wilmington, MA 01887 USA; tel. 978-6614568, fax 978-988-8798, and e-mail bgu@ gsig.com.

\section{Henry Helvajian is a} senior scientist at Aerospace Corp., a nonprofit organization created by the U.S. Congress with the goal of providing research and development in the area of space systems development. He has worked on research problems in the area of gas-phase photochemistry and kinetics of activated radical species and on photophysical processes of low-fluence laser-material interaction phenomena. Helvajian's present research interest is in the area of controlled fluence laser processing for selective activation of materials property changes with site-selective control. He has been working on developing a materials processing technique for non-ablative and true 3D patterning of photostructurable glass ceramic materials. His research group is focused on developing materials processing techniques that will enable the mass-producible and mass-customizable manufacturing of nanosatellites primarily

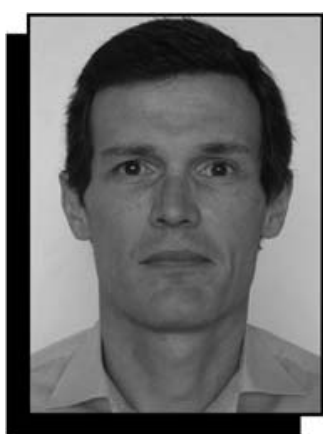

Andrew Holmes

composed of glass ceramic materials.

Helvajian can be reached at the Micro/ Nanotechnology Department, Space Materials Laboratory, Aerospace Corp., 2350 E. El Segundo Blvd., El Segundo, CA 90245 USA; tel. 310-3367621, fax 310-563-3175, and e-mail henry. helvajian@aero.org.

Andrew Holmes is a reader in microelectromechanical systems (MEMS) in the Department of Electrical \& Electronic Engineering at Imperial College London. He received his BA degree in natural sciences from the University of Cambridge in 1987 and his PhD degree in electrical engineering from Imperial College London in 1992

Holmes has worked on a wide range of topics in optical signal processing, integrated optics, and MEMS, and has published approximately 90 papers in these areas. He is a cofounder and director of Microsaic Systems Ltd., an Imperial College spinout company started in 2001 to exploit the college's MEMS research. His current research interests lie in the areas of micropower generation, MEMS devices for microwave applications, and laser processing for MEMS manufacturing.

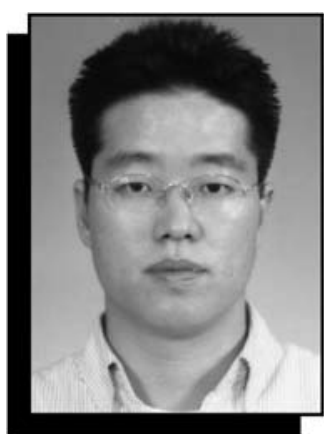

David J. Hwang

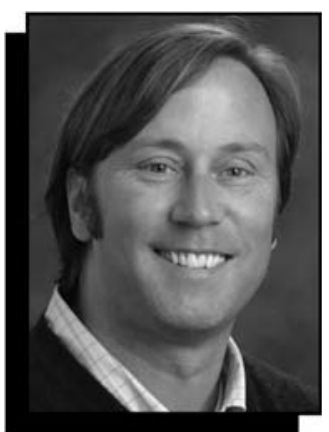

Frank E. Livingston
Holmes can be reached at the Department of Electrical and Electronic Engineering, Imperial College London, Exhibition Road, London SW7 2AZ, UK; tel. 44-207594-6239, fax 44-207594-6308, and e-mail a.holmes@ imperial.ac.uk.

David J. Hwang is a postdoctoral research associate in the Mechanical Engineering Department at the University of California, Berkeley. He received his BSE and MSE degrees in mechanical engineering from Seoul National University in 1995 and 1997, respectively, and his $\mathrm{PhD}$ degree in mechanical engineering from UC-Berkeley in 2005.

Hwang worked for the Samsung Advanced Institute of Technology (SAIT) in South Korea from 1997 to 2000 , where he was involved in automotive engine control, superconductorrelated cryogenics, and microjetting devices. His major research interest is in micro/nanoscale processing of electronic materials based on pulsed lasers coupled through high-magnification objective lenses and/or optical nearfield probes.

Hwang can be reached at 5144 Etcheverry Hall, University of California,
Berkeley, CA 94720-1740

USA; tel. 510-642-1006 and e-mail djhmech@ me.berkeley.edu.

Frank E. Livingston is a research scientist in the Micro/Nanotechnology Department of the Space Materials Laboratory at Aerospace Corp. He received his BA degree from Claremont McKenna College and his MS degree from California State University at Fullerton, both in chemistry. At Cal State with B.J. Finlayson-Pitts, studying the kinetics and mechanisms of heterogeneous processes relevant to the tropospheric chemistry of polluted marine regions and ozone destruction in the Arctic.

Livingston earned his $\mathrm{PhD}$ degree in chemistry from the University of California, Los Angeles, where he studied microscopic surface chemistry and photoionization spectroscopy of alkali halide clusters and nanocrystals under the direction of R.L. Whetten He then accepted a senior postdoctoral research fellowship with S.M. George at the University of Colorado, Boulder, where he developed several novel laser-based techniques to study kinetic diffusion processes on molecular solids. Fullerton, he worked 


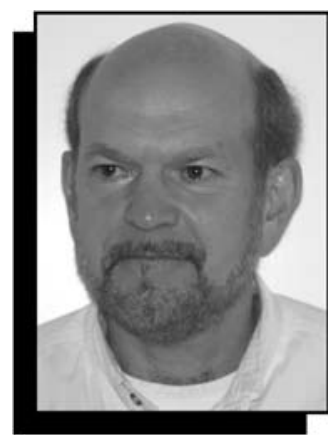

Doug A.A. Ohlberg

Livingston's current research focuses on the photophysics and chemistry of laser-material interactions, laser processing of photosensitive glass ceramics and protean materials, ultrafast optical spectroscopy of laser-surface phenomena, and optical trapping and nanoparticle ensemble assembly for nanostructured materials development. He has a keen interest in the laser-induced transformation of materials, for example, phase and chemical transformations of multimetallic perovskite and gallium oxide nanoparticle thin films for the development of improvedsensitivity uncooled IR detectors and electroluminescent phosphors for flexible displays. He has authored 45 peer-reviewed papers and book chapters.

Livingston can be reached at the Micro/ Nanotechnology Department, Space Materials Laboratory, Aerospace Corp., 2350 E. El Segundo Blvd., El Segundo, CA 90245 USA; tel. 310-3360056, fax 310-336-5846, and e-mail frank.e. livingston@aero.org.

Kurt Mueller is a member of the technical staff at Max-Planck-Institut für Biophysikalische Chemie. He can be reached at MPI für

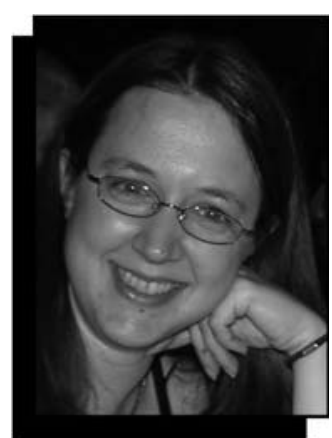

Rachel Oliver

Biophysikalische Chemie, Am Fassberg 11, D-37077 Göttingen, Germany; and by e-mail via mstuke@gwdg.de.

Torsten Mueller earned his PhD degree in biophysics at Humboldt University in Berlin. He can be reached at Evotec AG, c/o HumboldtUniversität zu Berlin, Invalidenstrasse 42, D-10115 Berlin, Germany; and by e-mail via mstuke@gwdg.de.

Doug A.A. Ohlberg is a member of the Quantum Structures Research Initiative group at Hewlett-Packard (HP) Laboratories. He received his BS degree in chemistry in 1989 from California State University, Fresno. Ohlberg's MS degree in chemistry was earned in 1995 at the University of California, Los Angeles, with R. Stanley Williams as his advisor. From 1994 to 1996, Ohlberg worked as a graduate fellow for Sandia National Laboratories in their Flat-Panel Display Initiative group.

Ohlberg joined HP

Laboratories in 1996.

His early research efforts at $\mathrm{HP}$ involved the formation of selfassembled nanostructures, including germanium silicide nanodots and rare-earth silicide nanowires. He is

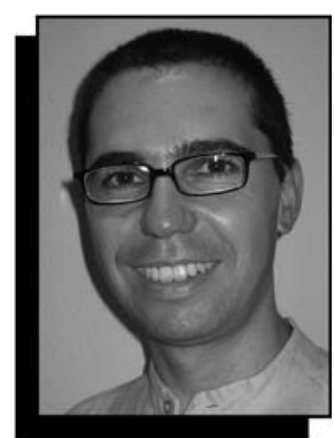

Pere Serra

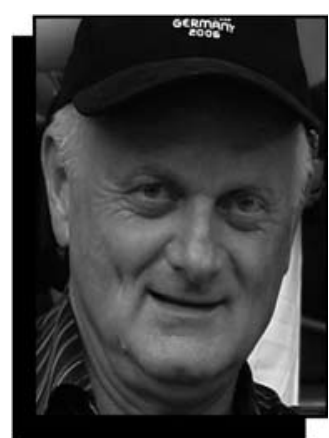

Michael Stuke

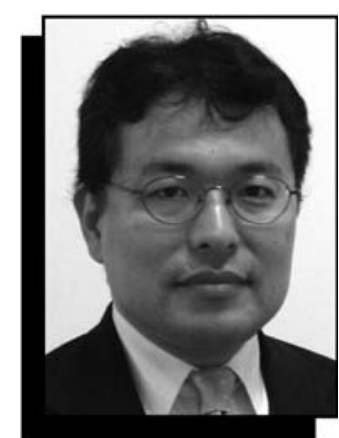

Koji Sugioka currently investigating the growth and electrical behavior of various materials systems for implementation as switching elements in the crossbar architecture that HP is developing for nanoscale logic and memory circuitry.

Ohlberg can be reached at Hewlett-

Packard Labs, 1501 Page Mill Rd., MS1123, Palo Alto, CA 94304; tel. 650857-8487, fax 650-2369885, and e-mail doug_ohlberg@hp.com.

Rachel Oliver is a research fellow in the Department of Materials Science and Metallurgy at the University of Cambridge. She obtained her doctorate from Oxford University in 2003 . Her doctoral training included periods as an intern at Hewlett-Packard Laboratories in Palo Alto, California. Oliver's research interests center on nanoscale structures in optoelectronic materials.

Oliver can be reached at the University of Cambridge, Department of Materials Science and Metallurgy, New Museums Site, Pembroke Street, Cambridge, CB2 3QZ, UK; e-mail rao28@cam.ac.uk.

Pere Serra is an associate professor at the University of Barcelona. He obtained his $\mathrm{PhD}$ degree at the university in 1997, where he investigated the expansion dynamics of laser-generated plasmas of diverse materials. In particular, Serra devoted much of his work to the study of bioactive ceramics for the production of medical implants through pulsed laser deposition. This research motivated him to turn his attention to another laser-based approach to the processing of materials for biomedical applications-laser surface treatment of biocompatible metals and ceramics. He is now engaged in the development of laser microfabrication techniques for the preparation of miniaturized biosensors and lab-on-a-chip systems.

Serra can be reached at the University of Barcelona, Applied Physics and Optics Department, Martí i Franquès $1, \mathrm{E}-08028$

Barcelona, Spain; e-mail pserra@ub.edu.

Michael Stuke is a senior scientist at the Max Planck Institute for Biophysical Chemistry in Göttingen, Germany, and professor of physics. He studied at the University of Marburg, Technische Universität Munich, and the University of Heidelberg. After receiving his $\mathrm{PhD}$ degree in physical chemistry, he became a staff member at MPI Göttingen, where he heads the Laser Chemical Processing of Materials group. He has worked at the University of Southern California, ENEA Frascati in Italy, RIKEN in Japan, and Hewlett-Packard Laboratories in Palo Alto, California. Stuke received the Otto Hahn Medal from the Max Planck Society and is editor in chief of Applied Physics A, Materials:

Science and Processing.

Stuke can be reached at Max-Planck-Institut für Biophysikalische Chemie, PO Box 2841, D-37018 Göttingen, Germany; e-mail mstuke@gwdg.de.

Koji Sugioka is a senior research scientist at RIKEN (the Institute of Physical and Chemical Research) and a guest professor at Tokyo University of Science and Tokyo Denki University. He received $\mathrm{BE}, \mathrm{ME}$, and $\mathrm{PhD}$ degrees in electronics from Waseda University in 1984, 1986, and 1993, respectively. He joined RIKEN in 1986.

At RIKEN, he has worked on doping, etching, and deposition of semiconductors and surface modification of metals using excimer lasers. Sugioka also studied the microfabrication of hard 


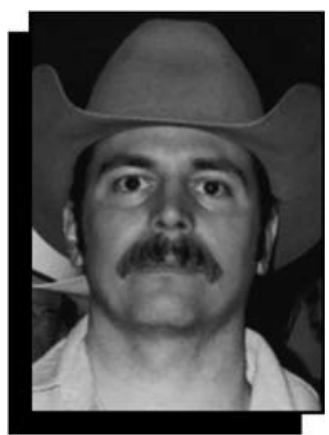

Kirk Williams

materials such as glass by using VUV and ultrafast lasers. His current interests center on the development of advanced laser microprocessing techniques for performing surface and 3D microstructuring of transparent materials, with applications to lab-ona-chip and photonic devices.

Sugioka has received several awards for his

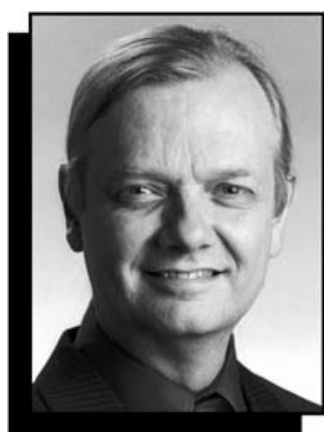

R. Stanley Williams

research and inventions in the area of laser microprocessing. He has published more than 100 articles, given more than 40 invited talks at international conferences and more than 50 invited talks at domestic conferences, and holds over 20 patents or pending patents. He has served as a conference chair, co-chair, and committee member for numerous international conferences. He is also editor in chief of the Journal of Laser Micro/ Nanoengineering.

Sugioka can be reached at Laser Technology Laboratory, RIKEN, Wako, Saitama 351-0198, Japan; tel. 8148-467-9495, fax 81-48462-4682, and e-mail ksugioka@riken.jp.

Kirk Williams is senior $R \& D$ engineer and part owner of Free Form Fibers LLC in Plattsburgh, New York. He received his BSc degree in mechanical engineering in 1998 and MSc degree in manufacturing systems engineering in 2000 from Louisiana Tech University. In March 2006, he received his $\mathrm{PhD}$ degree in microsystems engineering from the Ångström Space Technology Center at Upp- sala University in Sweden. He took brief pauses from his academic studies to work in the Department of Materials Chemistry at Uppsala in 1998 and at the Max Planck Institute for Biophysical Chemistry in Göttingen, Germany, in 2000.

His research is focused on the production and characterization of LCVD-deposited microstructures such as fibers, microcoil heaters for microthrusters in space, and mass spectrometry. At Free Form Fibers, William's work is focused on using LCVD to deposit exotic and specialty material fibers for commercial use.

Williams can be reached by e-mail at kirk@kirk-williams.nu.

R. Stanley Williams is a senior HP fellow at
Hewlett-Packard Laboratories and founding director (since 1995) of the Quantum Science Research group. He received a BA degree in chemical physics in 1974 from Rice University and his PhD degree in physical chemistry in 1978 from the University of California, Berkeley. Williams was a faculty member of the Chemistry Department at UCLA from 1980 to 1995. His primary scientific research during the past 20 years has been in the areas of solid-state chemistry and physics.

Williams can be reached at Quantum Structures Research, Hewlett-Packard Laboratories, 1501 Page Mill Rd., Palo Alto, CA 94304 USA; e-mail stan. williams@hp.com.

\section{The Materiabls Gateway-www.mrs.org}

\section{Advertisers in This Issue}

Page No.

\begin{tabular}{lllr}
\hline Carl Zeiss SMT, Inc. & www.smt.zeiss.com/nts & info-usa@smt.zeiss.com & 56 \\
\hline High Voltage Engineering Europa B.V. & www.highvolteng.com & info@highvolteng.com & Inside front cover \\
\hline Huntington Mechanical Laboratories, Inc. & www.huntvac.com & vacman@huntvac.com & Outside back cover \\
\hline Janis Research Company, Inc. & www.janis.com & sales@janis.com & 39 \\
\hline Kurt J. Lesker Company & www.lesker.com & materialsgroup@lesker.com & Inside back cover \\
\hline National Electrostatics Corp. & www.pelletron.com & nec@pelletron.com & 63 \\
\hline Penguin Group (USA) & www.penguin.com/academic & academic@us.penguingroup.com & 46 \\
\hline Shiva Technologies, Inc. & www.shivatec.com & sales@shivatec.com & 54 \\
\hline Society of Vacuum Coaters & www.svc.org & svcinfo@svc.org & 80 \\
\hline Veeco Instruments, Inc. & www.veeco.com/caliber & & 55 \\
\hline
\end{tabular}

